\title{
The ATLAS Experiment Entering into Operation: Overview, Motivation and Status of the Project
}

\author{
P. Jenni
}

The Large Hadron Collider LHC at the European Laboratory for Particle Physics CERN near Geneva will deliver particle collisions at the highest energy ever achieved in a laboratory. After more than 15 years of design and construction efforts, the LHC and its experiments are finally starting operation. Besides the giant accelerator, which is installed in a ring tunnel 27 $\mathrm{km}$ in length about $100 \mathrm{~m}$ underground, the no less impressive and complex detectors are ready for data collection.

A general overview of ATLAS, one of the key experiments, has been given. The LHC will allow ATLAS to study for the first time fundamental physical phenomena as they occurred very shortly after the Big Bang. ATLAS will address questions like: Why do particles have a mass, What is the non-visible dark matter in the Universe, Are there more than four dimensions in Nature, and What are the smallest building blocks of matter? The expectations for new discoveries are high, since physicists have for decades been eagerly awaiting this exploratory step into the unknown.

The technically sophisticated and highly complex ATLAS detector has been developed and constructed through world-wide collaboration, and the Czech teams have made remarkable contributions to the project since its conception some 20 years ago, very much so thanks to the constant strong support and encouragement provided by Professor Niederle. One important and most pleasant milestone in the history of ATLAS was the yearly Overview Week. The
September 2003 overview took place in Prague, and was opened with an address by Professor Niederle.

Some highlights of the construction and commissioning of the detector have been illustrated, as well as several examples of anticipated discovery signals. It would be artificial and almost impossible to attempt to summarize the huge amount of work done by the collaborating teams within just a few pages. The reader is therefore referred to the two following references. A comprehensive and detailed description of the construction of the ATLAS detector and its expected performance can be found in Ref. [1], while Ref. [2] is part of an overall LHC project publication that provides an account of the ATLAS experiment as a whole.

\section{References}

[1] Aad, G., et al.: The ATLAS Collaboration, 'The ATLAS Experiment at the Large Hadron Collider', JINST 3 (2008), S08003.

[2] Jenni, P.: In: The Large Hadron Collider: a Marvel of Technology, edited by L. Evans, EPFL Press, 2009, p. 182-199. ISBN 978-2-940222-34-2.

Peter Jenni

CERN, former Spokesperson

of the ATLAS Collaboration

European Organization for Nuclear Research CERN CH-1211, Genève 23, Switzerland 